\title{
Microsensors for Mars: Trace Analyte Detection in a Simulated Martian Environment
}

\author{
$\underline{\text { Kurt D. Benkstein }}{ }^{1}$, Philip H. Rogers ${ }^{1}$, Christopher B. Montgomery ${ }^{1}$, Jerry Jin ${ }^{2}$, Baranidharan Raman ${ }^{2}$, \\ Steve Semancik \\ ${ }^{1}$ National Institute of Standards and Technology, Material Measurement Laboratory, \\ 100 Bureau Dr., MS 8362, Gaithersburg, MD 20899-8362, USA \\ kurt.benkstein@nist.gov \\ ${ }^{2}$ Department of Biomedical Engineering, Washington University, St. Louis, MO 63130, USA
}

\begin{abstract}
:
Chemiresistive microsensor arrays are being developed and tested in a simulated Martian environment. Target analyte species include trace small molecules that may indicate current geological or, potentially, biological activity on Mars. The sensing films are based upon robust, nanostructured metal oxide materials, including doped and undoped tin oxide, tungsten oxide and indium oxide. In combination with dynamic operating temperatures and advanced signal processing, we show in this presentation the capabilities of the microsensor arrays for analyte discrimination and quantification at target levels below $500 \mathrm{nmol} / \mathrm{mol}$ in a simulated Martian environment. The simulated environment uses a carbon dioxide-rich background and low temperature and pressure.
\end{abstract}

Key words: chemical microsensor, gas-phase sensing, sensor arrays, Mars

\section{Introduction}

The identification of trace gases in the Martian atmosphere is an important component of understanding current geological and, potentially, biological processes. Methane has been detected spectroscopically in the atmosphere at trace concentrations (tens of $\mathrm{nmol} / \mathrm{mol}$ ) [1], but the source is under debate. Identifying point sources of the gas along with geographically associated chemical species may help to determine whether the source of the gas is biological or geological. We are developing and evaluating small, low-power microsensor arrays [2] and associated signal analyses as a screening tool for the detection of trace analytes of interest (e.g., hydrogen, methane, ethane and sulfur dioxide). The sensors consist of an array of temperatureprogrammable platforms (microhotplates) coated with chemiresistive sensing films. We infer the gas-phase chemical composition of sample gas streams by analysis of the temporal variation of the sensing-film electrical resistances. The challenges for this study include the low concentrations of analytes, the low oxygen content of the atmosphere (compared to the terrestrial atmosphere) and the low atmospheric pressure in general (Mars $P_{\text {avg }}=0.6 \mathrm{kPa}$ ), and the low temperature (Mars $\left.T_{\text {avg }}=210 \mathrm{~K}\right)[3]$.

\section{Materials and Methods}

Select sensors from a 16-element chemical microsensor array were used in these studies. Resistance measurements of the sensing films were made through interdigitated Pt surface contacts on the microhotplate platforms (100 $\mu \mathrm{m}$ across) [2]. All sensing films were metal oxide semiconductors, and were deposited onto microhotplate platforms from varied solutions/dispersions by microcapillary pipetting [4]. The Martian environment was simulated chemically $(0.15 \%$ oxygen with the balance as carbon dioxide), as well as physically $\left(P_{\text {cell }} \approx 0.8 \mathrm{kPa}, T_{\text {cell }} \approx 200 \mathrm{~K}\right)$. The low pressure was maintained while the sample stream was held at a steady $100 \mathrm{~mL} / \mathrm{min}$ flow rate using a throttled pump. The sample cell and gas flow lines were immersed in a dry ice/methanol bath to achieve the low temperature, which was monitored in the cell using calibrated platinum resistance thermometers. The chemiresistor arrays were exposed to target analytes from $20 \mathrm{nmol} / \mathrm{mol}$ to $450 \mathrm{nmol} / \mathrm{mol}$, both singly and in mixtures. Signals were modulated by pulsing the operating temperatures of the microsensors from $230 \mathrm{~K}$ to $730 \mathrm{~K}(\Delta T=50 \mathrm{~K}$ per step). Futhermore, each temperature program used three different base temperatures to which the sensor element returned between pulses. A sensor resistance measurement was made at 
each temperature step (pulse and base). Data analyses, including linear discriminant analysis (LDA) and parallel factor analysis (PARAFAC), were performed to evaluate the capabilities of the array for analyte discrimination and quantification.

\section{Results and Discussion}

Figure 1 shows the testing chamber (the cell) used to simulate the Martian background and environment. Note the coiled delivery line at the bottom, which allows for cooling of the gas stream by the dry ice/methanol bath before it enters the sample cell. The ambient temperature and pressure used for these studies fall within the normal range observed on Mars [3]. Over the course of a 6 hour experiment, the temperature typically ranged from $205 \mathrm{~K}$ to $199 \mathrm{~K}$.

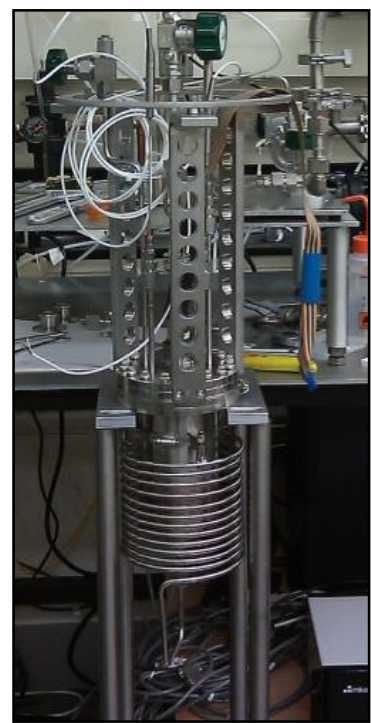

Fig. 1. The test cell for simulating the Martian background.

Figure 2 shows a microsensor element in the 16-element sensor array. Each element was coated with a metal oxide sensing film deposited using semi-automated capillary pipetting. The films were then thermally processed using the poly-silicon heaters embedded in the microsensor platforms. The sensing films used included tin oxide, antimonydoped tin oxide, tungsten oxide and indium oxide. Furthermore, films of titanium dioxide, iron oxide and copper oxide were also studied, but not used for the analysis described here.

Figure 3 shows resistance measurements (top panel) from a sensor element operating under simulated Martian conditions (background gas, temperature and pressure) upon exposure to four target analytes at trace concentrations. Microsensors were operated in a modulated temperature approach, which was tuned for the sensing material. An example temperature program for an indium oxide film is shown in Figure 3, bottom panel. In addition to the sequential exposure program shown in Figure 3 , microsensor arrays were also exposed to random mixtures of the analytes (two or three analytes at a time) to evaluate their capabilities for analyte discrimination and quantification.

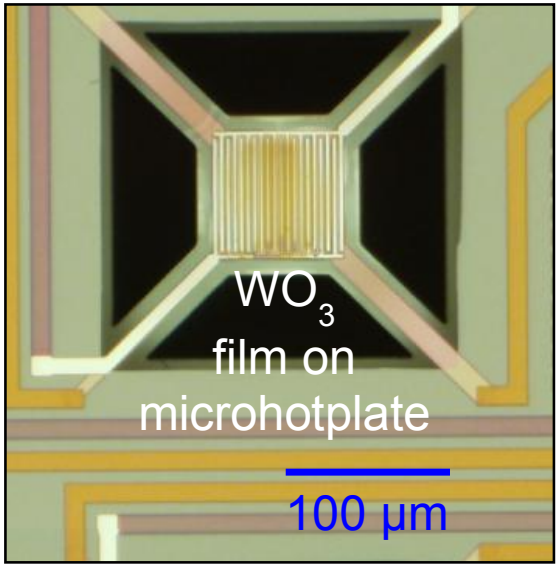

Fig. 2. A chemiresistive microsensor (one element of the array) with a tungsten oxide sensing film.

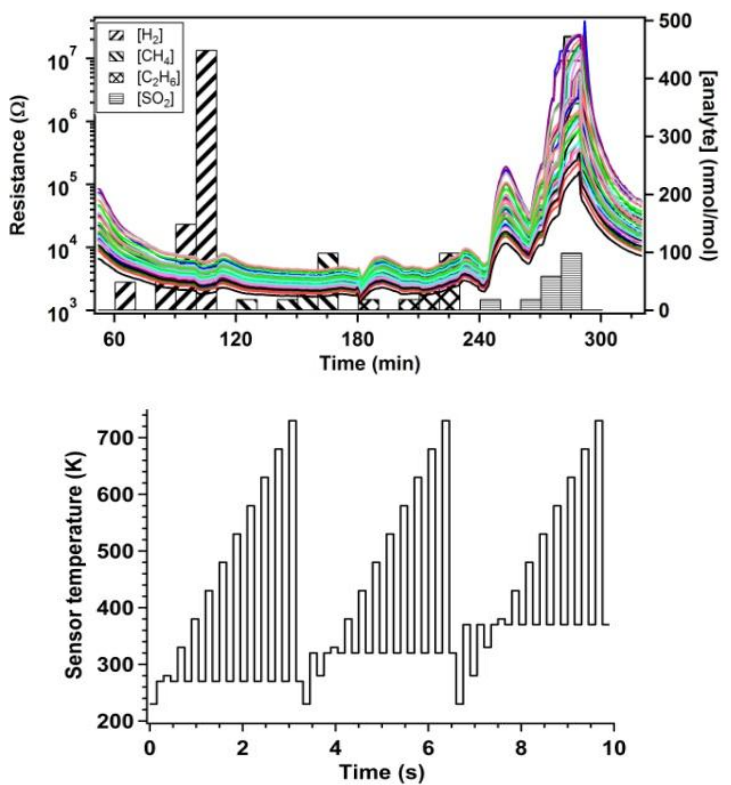

Fig. 3. Top Panel: Resistance data taken from the base temperatures (33 pseudo-isotherm data traces in total, see below) of a microhotplate chemiresistor using an indium oxide film during an analyte survey experiment. The bars indicate the presence and concentration (right axis) of target analytes. The spread between the traces is caused by the three different base temperatures and the thermal history before each measurement. Bottom Panel: The temperature program for this sensor element. Each pulse-temperature step corresponds to a pseudoisotherm trace above.

The data from the arrays were analyzed using several different approaches for both processing (e.g., background/drift compen- 
sation) and information extraction (i.e., analyte identification and quantification). The results from two approaches on data from multiple microsensor elements are shown in Figure 4. For the left panel, LDA is used to generate separation algorithms for three of the analytes. The right panel shows the separability of different analyte mixtures along two components extracted by PARAFAC along analyte dimension.

The sensor arrays were also evaluated for the ability to quantify the trace analytes in the mixtures. For example, Figure 5 shows the results of applying the LDA approach to the quantification of mixtures containing methane, sulfur dioxide and hydrogen. As seen in these histogram plots, the sensor array shows the capability to distinguish between the background and two concentrations of the analytes at concentrations of $200 \mathrm{nmol} / \mathrm{mol}$ and below. Each condition (the low and high concentration, as well as $0 \mathrm{nmol} / \mathrm{mol}$ of a particular analyte in Figure 5) also includes instances when one or both of the other analytes were present, as shown in the example flow program.
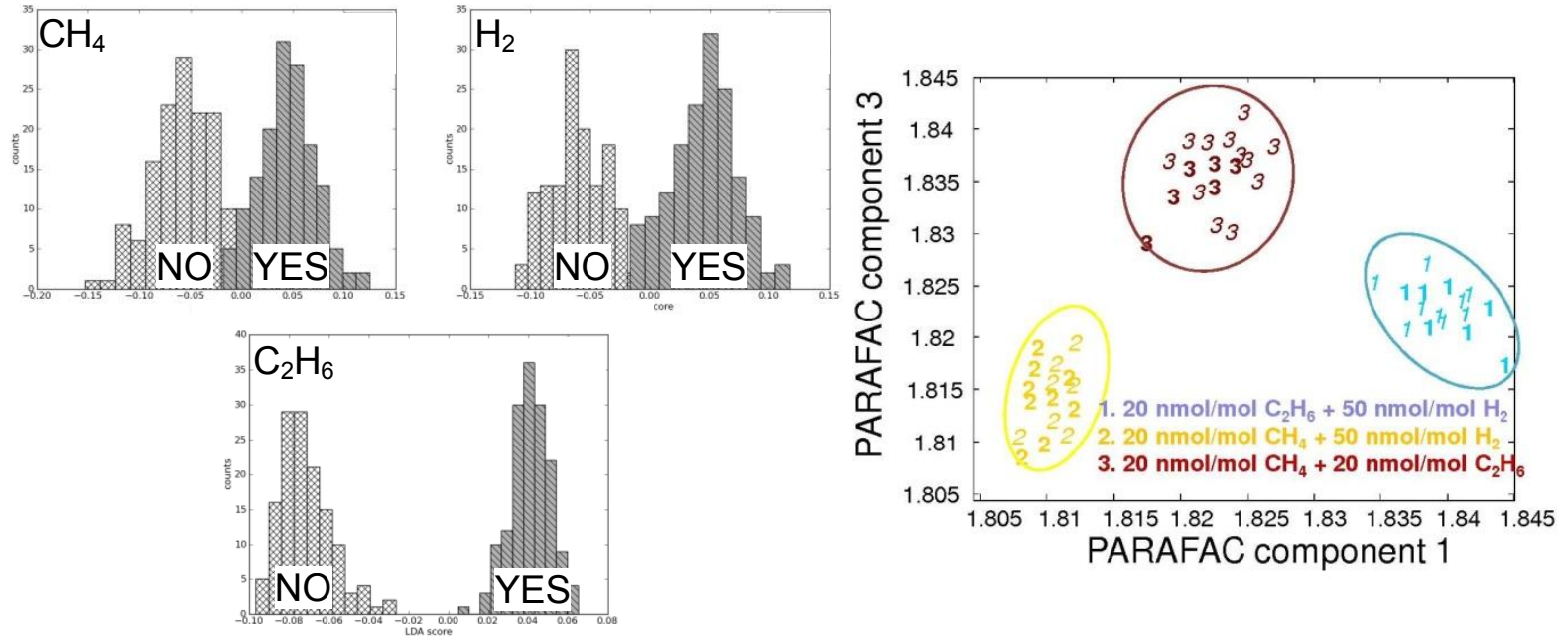

Fig. 4. Left panel: Histograms for discriminating between analytes in mixtures using LDA (bottom axes). Right panel: Parallel factor analysis of three-way data (analytes $\times$ temperatures $\times$ sensors) showing the discrimination of 3 binary mixtures. 

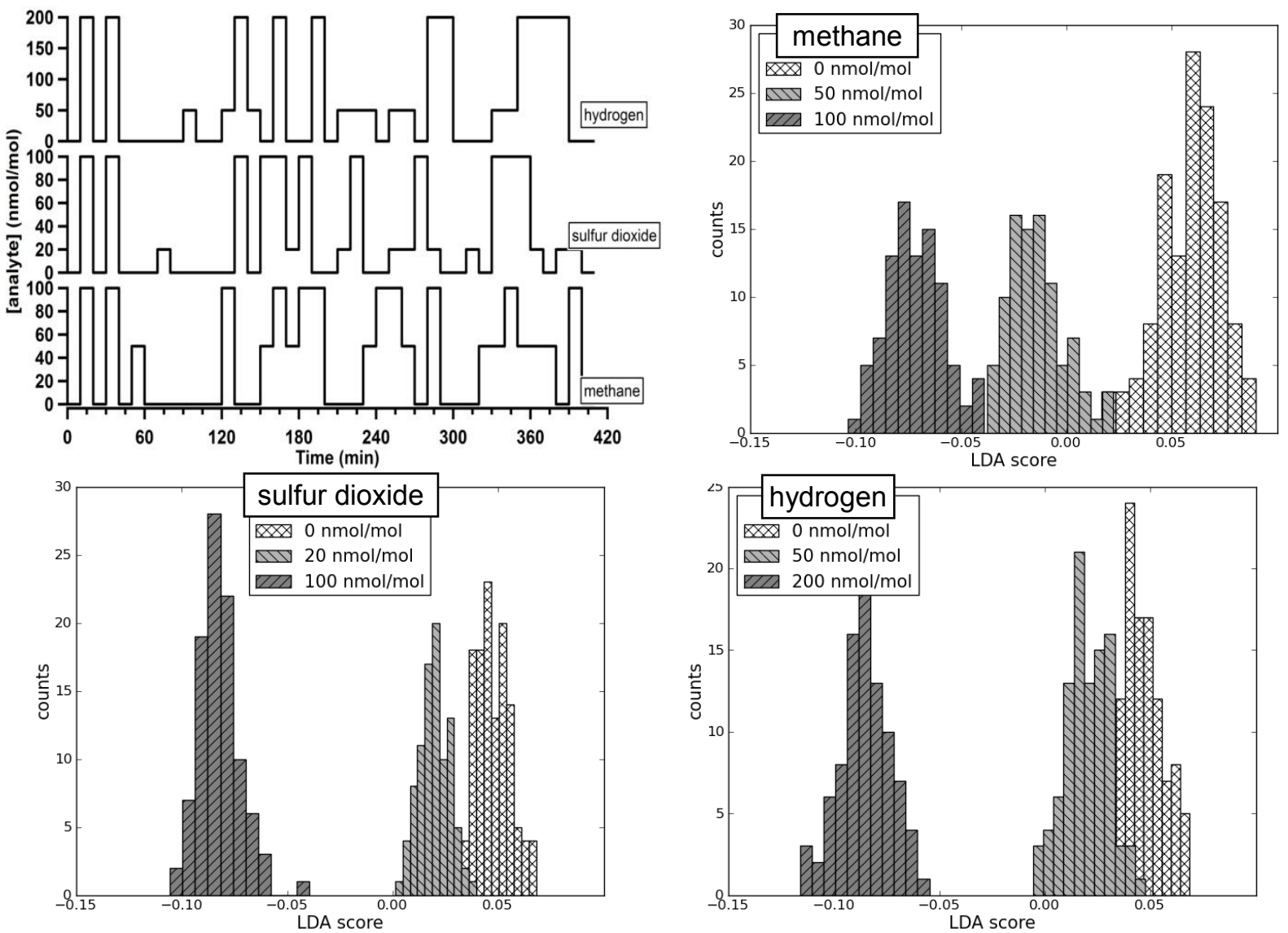

Fig. 5. Top Left Panel: Example program for delivering analyte mixtures to the sensor array. The results of LDAbased analysis for quantification are shown in the remaining panels as histograms. The three analytes (methane, hydrogen and sulfur dioxide, clockwise from the upper right panel) were each presented at two concentrations.

\section{Conclusion}

We have successfully demonstrated the viability of these microsensor arrays for the detection and quantification of mixed trace analytes in a simulated Martian background. The target analytes have been quantified at levels down to $20 \mathrm{nmol} / \mathrm{mol}$ in complex mixtures. Further optimization of the operational modes (materials and temperature programs) and signal analysis approaches is currently underway to improve the quantification of analytes in complex mixtures and to mitigate the effects of long-term baseline drift on sensor array performance.

\section{Acknowledgements}

We gratefully acknowledge funding for this research from NASA ROSES2009 PIDDP. PHR was supported by a National Institute of Standards Postdoctoral Associateship Award administered through the National Research Council.

\section{References}

[1] V. Formisano, S. Atreya, T. Encrenaz, N. Ignatiev, M. Giuranna, Detection of Methane in the Atmosphere of Mars, Science 306, 17581761 (2004); doi: 10.1126/science.1101732

[2] K.D. Benkstein, C.J. Martinez, G. Li, D.C. Meier, C.B. Montgomery, S. Semancik, Integration of nanostructured materials with MEMS microhotplate platforms to enhance chemical sensor performance, J. Nanoparticle Res. 8, 809822 (2006); doi: 10.1007/s11051-005-9019-8

[3] Mars Fact Sheet, National Space Science Data Center. NASA.

(http://nssdc.gsfc.nasa.gov/planetary/factsheet/m arsfact.html)

[4] P.H. Rogers, S. Semancik, Development of optimization procedures for application-specific chemical sensing, Sens. Act. B. 163, 8-19 (2012); doi: 10.1016/j.snb.2011.11.015 